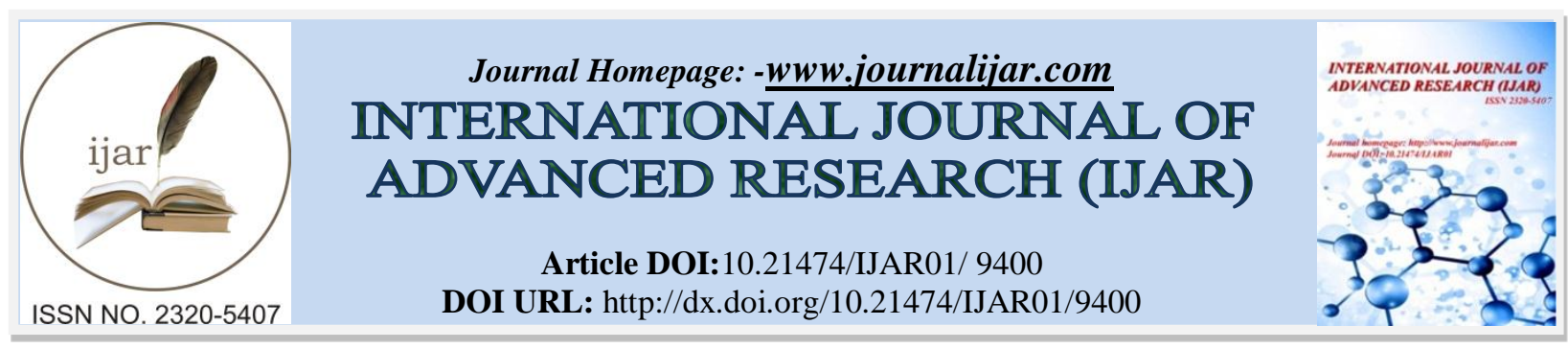

RESEARCH ARTICLE

\title{
UCHAMBUZI WA KISIMIOTIKI WA MISEMO YA KWENYE MAKAWA YA ZANZIBAR.
}

Maimuna Ahmed Makallo.

Idara ya Kiswahili na Lugha za Kigeni, Chuo Kikuu cha Taifa Zanzibar. (SUZA)

\section{Manuscript Info}

Manuscript History

Received: 17 May 2019

Final Accepted: 19 June 2019

Published: July 2019
Abstract

Copy Right, IJAR, 2019,. All rights reserved.

\section{Introduction:-}

Kawa kama kifaa ni ishara inayoashiria stara. Awali, umbo la kawa hulifanya kawa kukaa kwake iwe ni kufunika. Mkao huo wa kawa unasababisha kinachofunikwa na kawa kisionekani. Kufunikwa huko, humuachia mtu fumbo la kufumbua; kawa limefunika kitu au la. Na kama limefunika, limefunika nini? Pili, baadhi ya makawa huandikwa misemi. Misemo inayoandikwa katika makawa nayo hufunika maana zaidi ya misemo hiyo. Makala haya yamechunguza kawa na misemo ya kwenye makawa ili kujua maana ya kawa kama ishara, na maana ya ishara zilizomo katika misemo ya kwemye makawa. Uchunguzi huo ulifanywa kwa kutumia Nadharia ya Semiotiki inayojishughulisha na jinsi ishara na maana zinavyohusiana, zinavyotumiwa na zinavyofasiriwa (Resani, 2014). Aidha, Nadharia hiyo inaeleza kwamba, maana ya ishara haipatikani vitabuni wala mitamboni, bali hupatikana kwenye jamii (Chandler 1994). Hivyo, makala haya yametumia jamii ya Wazanzibari kupata maana ya kawa na maana ya ishara zilizotumika katika misemo ya kwenye makawa.

\section{Utangulizi}

Kawa ni kifaa kinachotumika kufunikia chakula, kupambia nyumba na kufanyiana mawasiliana katika jamii za Waswahili. Vifaa vingine vya ukili ni kama vile vipepeo, mikoba, vihangaisho. Watafiti kadhaa wamechunguza misemio inayoandikwa katika vifaa hivyo. Mlingi (2013) amechunguza dhima za misemo ya kwenye makawa, Mkonwa (2014) amechunguza jinsi mwanamke anavyoakisiwa katika misemo ya kwenye vipepeo, Salim (2015) amechunguza dhima za misemo ya kwenya mikoba. Hivyo, kuna haja sasa ya kuchunguza misemo pamoja na vifaa vinavyoandikwa misemo hiyo.

\section{Nadharia ya Semiotiki (NS)}

Semiotiki ni neno lililotohelewa kutoka kwenye neno lenye asili ya Kiyunani. Neno hili lina maana ya 'uhakiki wa ishara'. Kwa maana nyingine Semiotiki au Semiolojia ni taaluma inayojishughulisha na jinsi alama na maana zinavyohusiana, zinavyotumiwa na zinavyofasiriwa (Resani, 2014). Nadharia ya Semiotiki iliasisiwa tangu karne ya tano katika kipindi cha Hipocrates (Eschbach, 1983, Wanjiku 2006). Nadharia hii ilififia kwa muda mrefu hadi ilipoanza kutumiwa tena na Nietzsche (1883 - 1892). Baadaye Freud, Jung na Heidger waliiimarisha nadharia hiyo kwa kuanza kuitumia kutathmini uhusiano uliopo baina ya kitu cha kidhahania/halisia na alama za kiisimu na zisizo za kiisimu. Hadi kufikia miaka ya 70 ndipo uhakiki wa uashiriaji ulipopata uzito zaidi (Culler, 1981).

Nadharia hii iliimarishwa zaidi kwa kuwekewa mihimili na mhakiki Barthes (1964, 1977, 1981, 1984. 1986). Mhakiki huyo anasema kwamba kazi huhakikiwa kwa kugawanywa katika vipande vidogo vidogo. Vipande hivyo aliviita leksia. Aidha, Barthes anaeleza kwamba vipande hivyo hujenga misimbo au kanuni za kumuongoza mhakiki. Kwa mantiki hiyo Barthes aliunda misimbo ya aina tano ya ufasili wa ishara. 


\section{Msimbo wa Matukio:}

unashughulikia muundo wa kazi; tukio lipi limeanza na likafuata lipi. Na si suala la kufuatana kwa matukio tu, hasha. Bali ni jinsi tukio la kwanza lilivyosababisha kutokea kwa tukio la pili.

\section{Msimbo wa Kihemenitiki:}

Msimbo huu unahusu matendo ya binadamu. Kuna baadhi ya matendo ya binadamu hutendwa kiishara. Mathalan baba wa bi harusi kupeana mikono na bwana harusi wakati wa kufunga ndoa ni ishara inayoashiria makabidhiano (Taib, 2014).

\section{Msimbo wa Kisemu:}

aina hii ya uhakiki inashughulikia maana nasibishi za ishara. Ishara hizi hufananisha vitu au mambo kutokana na vile vinavyoonekana. Ishara hizi zaweza kuwa ni picha au fasili ya msomaji wa ishara. Mfano, kopa linaashiria moyo, kiti kinaashiria enzi, zabibu zinaashiria mke, ndege anaashiria mke/mpenzi,

\section{Msimbo wa ki-ishara:}

msimbo huu huhakiki kazi kwa kuangali jinsi jambo moja linavyoashiria kutokea kwa jambo jingine. Mfano mawingu yanaashiria mvua, dawa inaashiria tiba, msiba unaashiria huzuni.

\section{Msimbo wa Kirejelezi:}

Urejelezi katika msimbo huu ni kufasili kazi kwa kurejelea utamaduni na tajriba ya maisha ya jamii inayohusika na kazi inayohakikiwa. Hivyo, kiashiria kimoja kinaweza kikawa na maana tafauti kati ya jamii moja na nyingine kutokana na kwamba jamii hutofautiana kiutamaduni.

Ishara katika Semiotiki zaweza kuwa maneno, picha, sauti, harufu, ladha, vitendo au vitu. Ishara hizo huchunguzwa zimetumikaje, zina maana gani na zinafasiliwa vipi. Makala haya yametumia NS katika kufasilia maana za misemo iliyoandikwa kwenye makawa zilizobabe ishara ili kujua maana asili na maana matini ya misemo hiyo.

\section{Kawa}

Kwa mujibu wa Suleiman Almas, katika mtandao wa Zanzi News blog (2011), asili ya utengezwaji wa kawa ni kutoka katika maeneo ya Afrika, haswa kutoka sehemu za mwambao. Akifananua zaidi, amesema, ususi wa kawa, hauna tafauti na ususi wa mkoba, kikapu, mkeka, upepeo na mswala. Vyote huundwa kwa kutumia ukindu. Watengenezaji wa vifaa hivyo, aghlabu ni kina mama. Hata hivyo, makala haya yamegundua kwamba ususi wa makawa upo wa aina tatu; makawa ya ukindu, makawa ya mimea laini kama vile njukuti mbichi, makawa ya muwaa na makawa ya ukili.

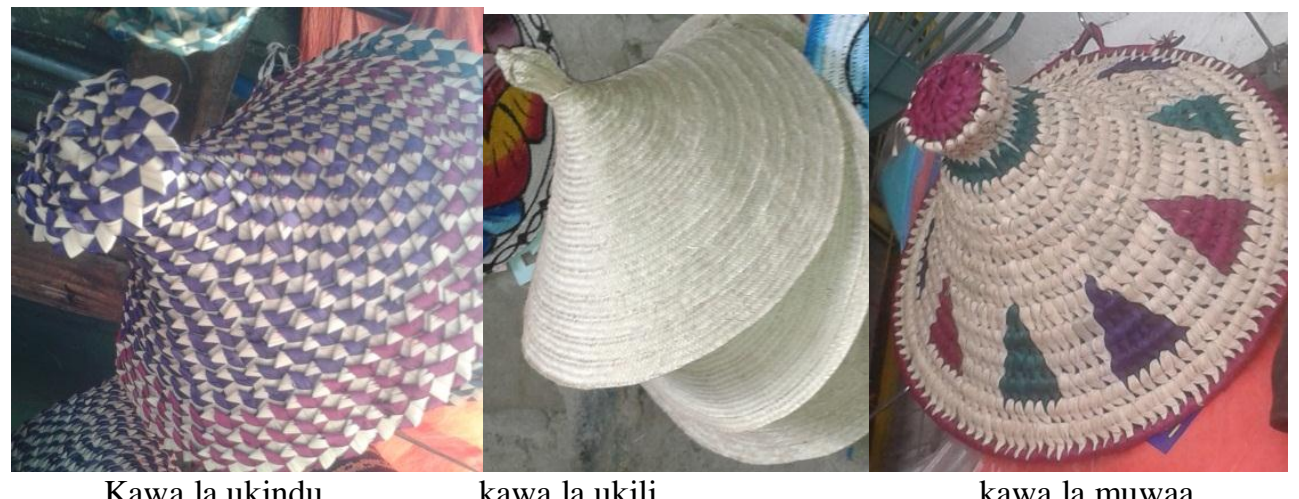

Hatua ya kwanza, katika ususi wa kawa, ni kuchana ukindu. Katika hatua hiyo, kwanza kabisa msusi huondoa ng'ongo. Hizo ni kingo ngumu za ukindu. Bila shaka, kazi yake ni kuuhifadhi ukindu usiharibike. Baada ya hapo, ukindu huchanwa katika chane/nyuzi nyembamba. Ukindu ukishachanwa hubadilika jina na kuwa ukili. 


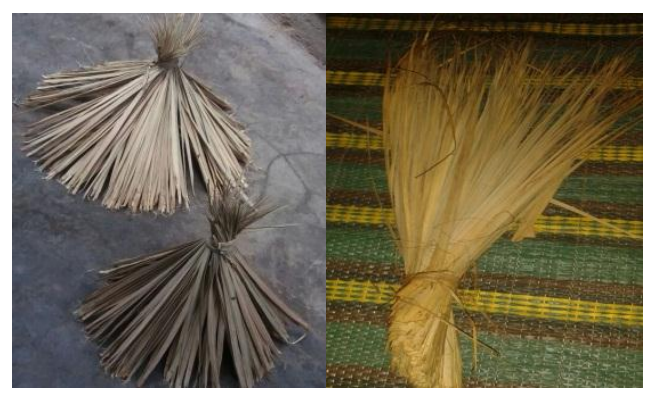

Ukindu

ukili

Ukili unapokuwa tayari, mpakasaji anaweza kuanza kuupakasa. Mpakasaji hupakasa urefu autakao kutengemeana na ukubwa wa kawa analotaka kulishona. Upakasaji huweza kuchukua siku tano hadi saba, kutegemeana na nafasi na wepesi wa mpakasaji. Baada ya hapo ukili huanikwa. Ukishakauka, hunyonyolewa ndasho. Hivyo, kuanikwa, ni kuufanya uwe mkavu na iwe rahisi kuunyonyoa ndasho zake.

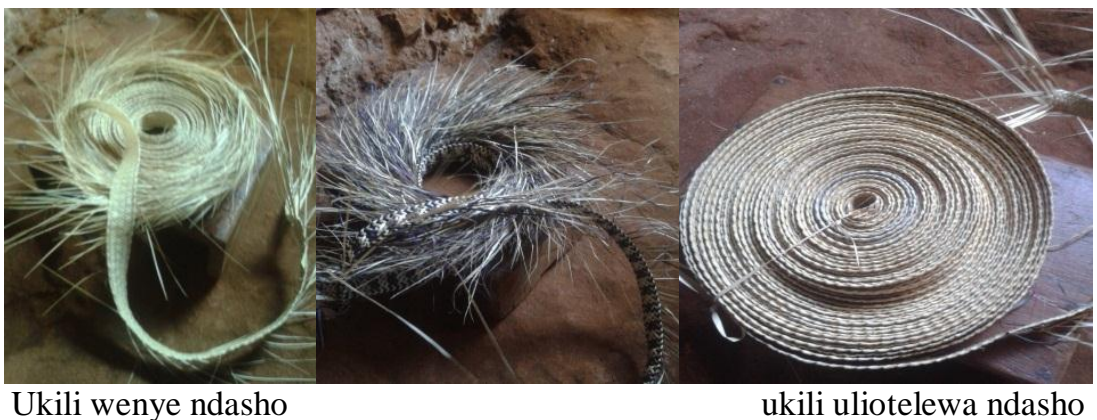

Hatua inayofuata, ni kushona kawa. Ushonaji huchukua siku moja au mbili. Shughuli hii nayo, pia yategemea nafasi na wepesi wa mshonaji. Hutokea wakati mwingine mpakasaji akawa mwingine na mshonaji akawa mwingine. Upakasaji si kazi ngumu sana kama ushonaji. Mshonaji akilikosea kawa, hutokea likawa kama bakuli.

Kawa huanzwa kushonwa kwenye kichwa (juu) na kushuka chini kwenye shingo. Huchanuliwa kidogokidogo, kadri linavyoendelea, kuelekea chini na kutengeneza umbo, la kama piramidi. Linapofika mwisho hushonwa kitu, kama kipua. Shingo na kichwa cha kawa hutumika kushikia kawa. Iwapo mtu anataka kufunika/kufunua kilichofunikwa, hushika sehemu hizo za kawa. Umbo la katikati, lililochanuliwa kuwa kama piramidi, ndilo linalositiri chakula kinapofunikwa kwa kawa. Iwapo kawa litanakshiwa kwa maua na/au kuandikwa semi, basi, huandikwa katika sehemu hiyo ya katikati iliyochanuliwa. Kipua cha kawa hutumika kutundikia kawa ukutani. Hivyo, semi iliyoandikwa kwenye kawa huweza kusomwa na yeyote aingiaye kwenye nyumba hiyo.

Kwa uasilia wake, kawa halihitaji kupakwa rangi au kuongeza urembo wa mauwa. Msusi kabla ya kuanza kuuchana ukindu huuchemsha kwa rangi maalumu kwa shughuli ya kubadilishia rangi, ukili. Zipo rangi za aina kadhaa; kijani, manjano, zambarau. Baadaye, huanika ukindu huo kwenye kivuli. Ukianikwa juani, huchujuka rangi yake. Ukishakauka huchanwa na hatimaye kuanza kupakaswa kwa kuchanganya kili zenye rangi tofauti tofauti. Kwa mpakasaji mzuri, hutumia rangi vizuri na hulifanya kawa kuvutia kwa muundo wake wa asili. (Tazama picha ya kawa hapa chini likiwa katika mtindo wa asili ).

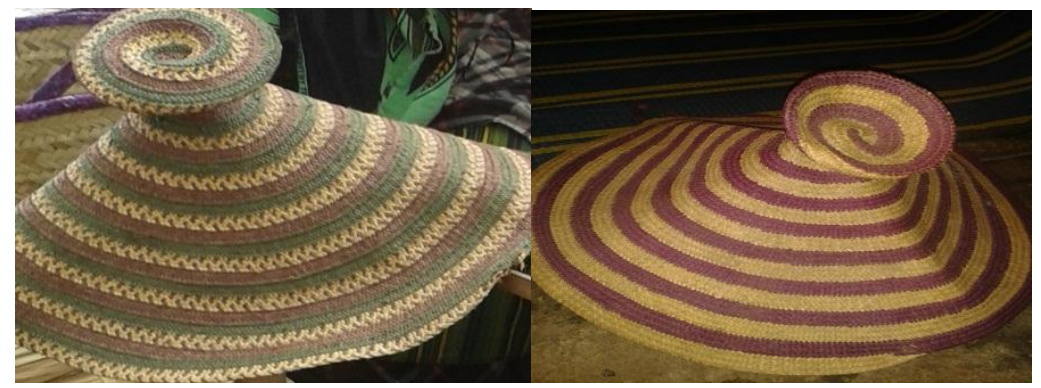




\section{Makawa yaliyohonwa katika mtindo wa asili}

Ama, aina nyingine ya mtindo wa makawa ni ule wa kulitia rangi kawa baada ya kushonwa. Kawa hupakwa rangi kwa kunakshiwa maua ya aina aina. Kawa, kabla ya kupakwa rangi, huwa na rangi yake nyeupe. Kisha, linachorwa maua kwa kutumia rangi. Baadaye, rangi nyeusi hupitishwa kufuatisha maua hayo. Picha za hapo chini, zinaonesha makawa kabla ya kuchorwa maua na hatua za mwanzo mwanzo za uchoraji maua (kabla ya maua kupitishiwa rangi nyeusi)

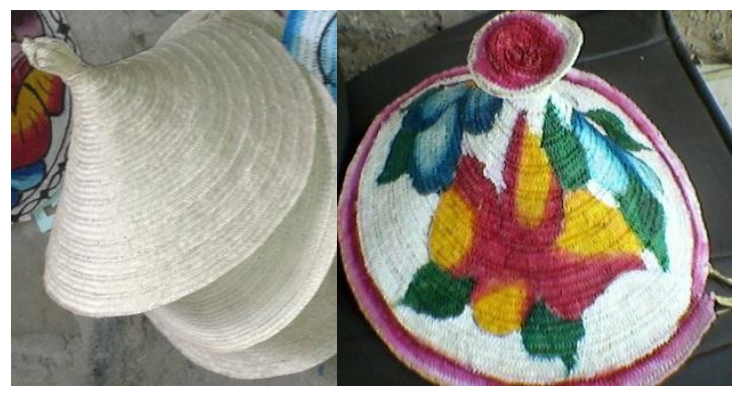

Chanzo: mtafiti (2017)

\section{Misemo ya kwenye Makawa}

Mbali na maua, wengine hupenda kuandikiwa misemo yenye ujumbe waliodhamiria kuufikisha kwa hadhira. Yawezekana, ujumbe ukawa unamkusudia mtu maalumu, au mtu yeyote atakayejaaliwa kulisoma kawa hilo. Misemo inayoandikwa katika makawa, huitwa Majina ya Makawa. Kawa hapa chini yamenakshiwa kwa rangi, maua na kuandikwa misemo.

\section{Chanzo: mtafiti (2018)}

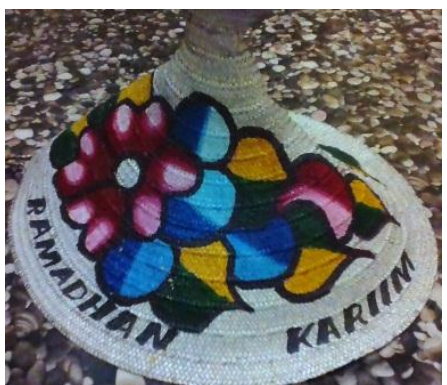

Kawa lililonakshiwa na kuandikwa semi

Aidha, kuna watumiaji wengine wa makawa wapendao kuchorewa maua tu, bila kuandikiwa semi yoyote. Kama linavyoonekanwa kawa la hapa chini.

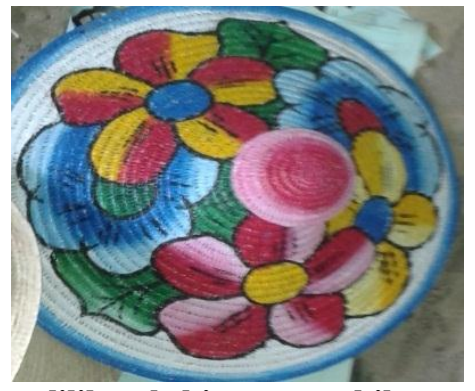

Kawa lililonakshiwa maua bila msemo

Chanzo: mtafiti (2018)

Mbali na hayo, makawa yatumiwayo sehemu za kazi au skuli, hayaandikwi semi yoyote. Semi za kwenye makawa zinahusu maisha ya wanajamii wawapo majumbani/mitaani mwao (Kipacha: http://researchgate.net/publication/286440186). Aidha, kawa la shule halipendezi kuandikwa, mfano elimu kwanza. Aidha, kuna misemo ya Makawa ambayo huandikwa katika lugha ya Kiarabu kwa kutumia hati za Kiarabu. Yapo pia, yanayoandikwa kwa lugha ya Kiarabu lakini kwa kutumia hati za Kilatini/Kiromani. Mfano wa makawa yaliyoandikwa hivyo, yanaonekanwa hapa chini. 


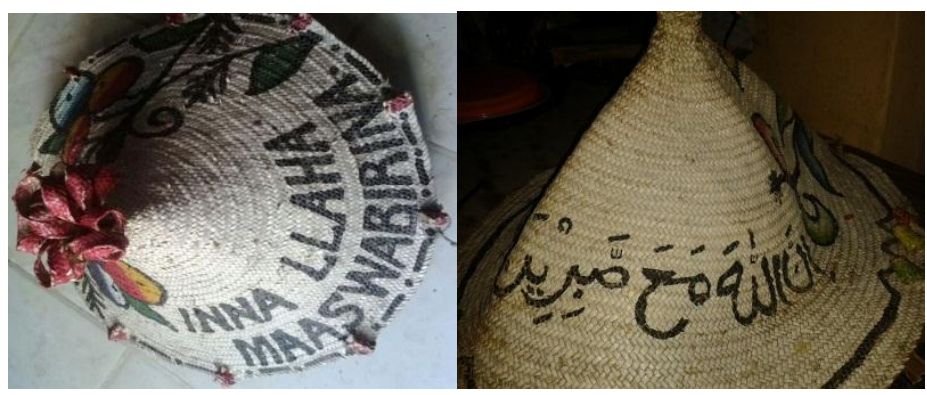

\section{Msemo wa Kiarabu katika}

msemo wa Kiarabau katika hati

\section{Hati za Kilatini}

za Kiarabu

Chanzo: mtafiti (2017)

Msemo wa kawa ulioandikwa kwa kutumia hati za kiarabu inasomeka "Inna Llaha maaswabiriina" kama inavyosomeka msemo ulioandikwa kwa hati za Kilatini/Kiruma.

\section{Uchambuzi wa Maana ya Kawa kama Ishara}

Maana ya kawa inapatikana kutokana na umbo lake na kazi zake. Msimbo ya nadharia ya Semiotiki ilitumika kubainidha maana za umbo na kazi za kawa ifuatavyo:

\section{Msimbo wa Ki -Ishara}

Kawa, ni ishara ya matukio kadhaa ya kijamii. Makawa yanaposhonwa kwa wingi yanaashiria kutokea kwa matukio kama vile arusi, Ramadhani na Maulidi. Misimu ya matukio hayo inapofika ndipo mafundi wa makawa hupata kazi nyingi za kupakasa, kushona na kuchora makawa.

Msimbo huu pia unaelekeza kwamba kawa linapokuwa mkekani au mezani huashiria kwamba chakula tayari. Wakati mwingine kawa limefunikwa tu, lakini kwa vile inajulikana kazi yake ni kufunika chakula, mlaji akiliona hujenga hamu ya kula. Aidha, kawa humvutia mlaji kufunua ili ajue kilichomo chini ya hifadhi hiyo. Na raha yake hasa, mlaji afunue yeye mwenyewe ili agundue kilichofunikwa. Hata hivyo, kufunua kawa, kwa mujibu wa Sengo (Darasani, 2018), kunategemea uhusiano wa aliyefunika na mfunuaji. Mume kwa mke ni haki na ni sawa. Si mtoto kwa mama. Si mgeni ugenini.

\section{Msimbo wa Kisemu}

Umbile la kawa hulifanya lihusianishwe na stara. Bi arusi anapofundwa hukabidhiwa makawa na kuambiwa akayatumie makawa hayo kuhifadhia chakula cha mumewe. Hivyo, bi arusi anafundishwa kujihifadhi kwa kutulia katika ndoa yake. Aidha, huambiwa kuwa kawa lina uwezo wa kufunika vyakula vingi kwa wakati mmoja kulingana na ukubwa na umbo la kifaa hicho. Uwezo huu wa kawa ni ishara ya kumtaka bi arusi awe na tabia ya kuhifadhi mambo yote ya nyumbani kwake.

Vilevile, kawa hutumika kuficha hali ya maisha. Mathalan, katika kipindi cha mfungo wa mwezi mtukufu wa Ramadhani, kawa hutumika sana kuficha, kwa tafsiri ya kuhifadhi futari, kabla ya kuwekwa wazi funguoni kwa mlo wa pamoja. Katika hali hiyo, watu wote wanakula chakula kimoja bila kujua nani kapika nini au nani leo hakuwa na cha kupika kabisa.

\section{Msimbo wa Kirejelezi}

Kawa vile vile ni ishara inayorejelea utamaduni wa watu wa mwambao wa Afrika Mashariki. Hivyo, kawa ni hifadhi ya mambo mema ya jamii ya Kizanzibari. Mbali na kawa, vyombo vingine vya asili kama chungu, mkungu, mtungi, mwiko, upawa, kung'uto, mbuzi na chano, vilikuwa na siri kubwa kwa matumizi ya kila mmoja, kwa jamii ya Kizanzibari.

Ama, kawa pia ni ishara ya pambo la nyumba kwa jamii ya Wazanzibari. Wazanzibari hutundika ukutani makawa yenye nakshi. Hii huifanya nyumba kuwa na haiba ya kupendeza. Kuna makawa mengine ambayo yapo kwa ajili ya mapambo tu. Makawa hayo huwa ni madogo kiumbo na mara nyingi huwa yameshikanishwa matatu kwa pamoja. Hivyo vikawa vya aina hii havitumiki kwa kufunikia chakula. 


\section{Chanzo: mtafiti (2018)}

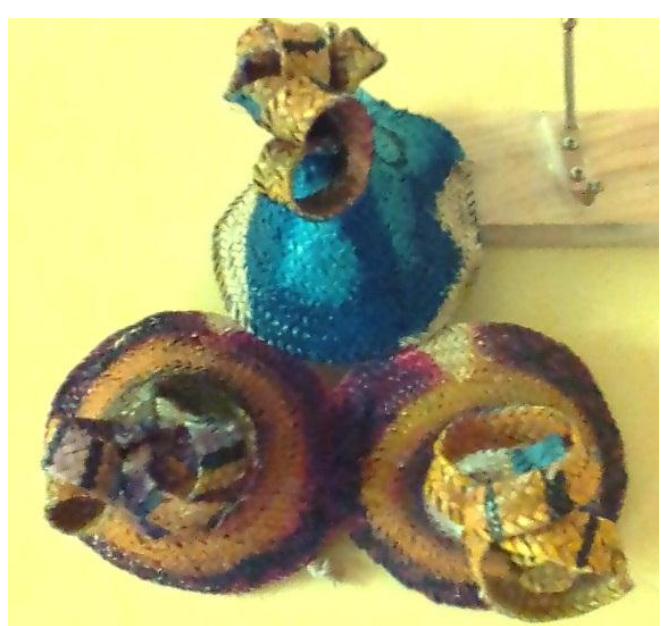

Vikawa vya mapambo

Makawa pia huashiria kutumwa kwa ujumbe kwa minajili ya kufanyia mawasiliano miongoni mwa wanajamii. Mtu anapoona kawa aghalabu huangalia limeandikwa nini. Inawezeka ujumbe wa kwenye kawa ukawa unamlenga mtu maalum au mtu yeyote. Kama ujumbe ni kwa mtu maalum naye anaweza kujibu kwa kutumia kawa vile vile. Aidha, mawasiliano hayo huwa yana dhamira maalumu. Dhamira hizo zaweza kuwa kurai, kuahidi, kuamrisha, kueleza, kusali (Buliba na wenzake 2006).

\section{Msimbo wa Kihemenetiki}

Tendo la kawa kukaa kwa kufunika hujenga aina nyingine ya stara; kumepikwa au hakujapikwa hakuna ajuwae. Hivyo, kiwepo cha kuliwa au kisiwepo sitara hiyo hubaki palepale. Mkao huu wa kawa umetokana na muundo wake. Ni nadra sana kuliona kawa limewekwa chali. Hivyo, umbo la kawa linasababisha kawa lifunikwe. Kufunikwa huko, humuachia mtu fumbo la kufumbua; kawa limefunika kitu au la. Na kama limefunika, limefunika nini?

Muundo wa kawa unafunika taarifa zaidi za maisha ya nyumba inayohusika. Kwa mantiki hiyo hiyo, misemo inayoandikwa katika makawa nayo hufunika maana zaidi. Kwa maneno mengine, makawa hutumika kama chombo cha mawasiliano. Mawasiliano ambayo kueleweka kwake kunategemea kueleweka kwa maana ya ujumbe uliowasilishwa. Mawasiliano miongoni mwa watu huongozwa na vigezo vya kitamaduni vya jamii inayohusika. Kwa maana hiyo, mawasiliano yanafahamika zaidi kwa kurejelea maisha ya jamii (Bathes 1986, Beck 2001, Taib 2014).

\section{Uchambuzi wa Maana za Misemo ya kwenye Makawa}

Misemo iliyochambuliwa iligawanywa katika makundi kwa kutumia kigezo cha msimbo. Uchambuzi ulikuwa hivi ifuatavyo:

\section{Msimbo wa Kisemu}

Kwa kutumia maana nasibishi za ishara, makala imebaini kwamba misemo iliyoingia katika msimbo huu ni haya yafuatayo:

1. Kula zabibu ulete majibu

2. Ndege tulia tunduni usinitie huzuni

3. Nina moyo sina jiwe mengine nipunguziwe

4. Heri kuishi na paka kuliko majirani wa hapa 


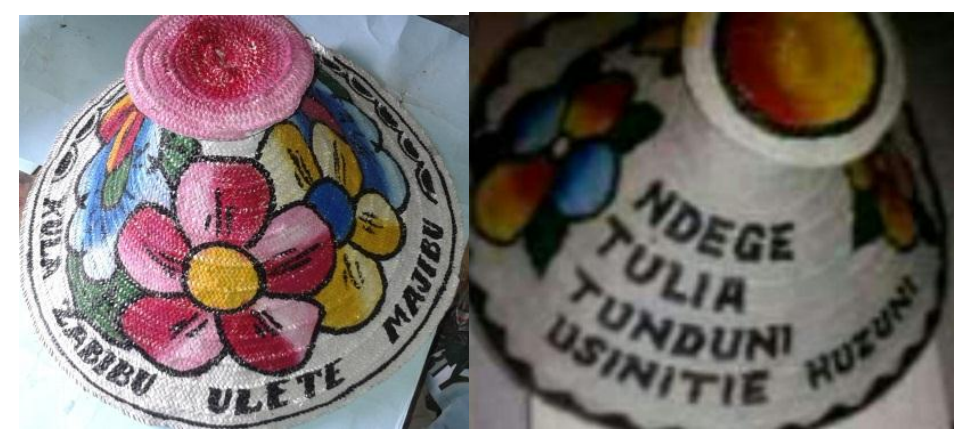

\section{Chanzo: Mtatifi (2016)}

Kwa maana ya asili, zabibu ni tunda. Tunda hilo limeumbwa kwa mkusanyiko wa vijitunda vidogovidogo, vilivyoshikiliwa kwenye kikonyo kimoja. Muundo wake huo hulifanya tunda hilo liwe na haiba ya kuvutia. Aidha, vijitunda vyake vina majimaji matamu yenye harufu nzuri. Kwa kutumia msinbo wa Kisemu wa NS unaoeleza kwamba maana nasibu yaweza kupatikana kutokana na vile ishara inavyoonekanwa (Barthes 1986, Taib 2014). Hivyo, kutokana na uzuri huo, tunda hilo laweza kunasibishwa na wema na/ au mapenzi.

Hivyo,katika msemo (1) hapo juu kula zabibu ina maana nakupenda. Na ulete majibu ni matokeo yanayotarajiwa yaletwe na mapenzi. Kwa mfano mapenzi huzaa ndoa, ndoa nayo hutarajiwa kuleta kizazi na kujenga maisha ya familia yenye kusikilizana. Hayo yote yakitokea familia itaishi kwa amani na furaha. Msemo huo, waweza pia kufasiliwa kama 'nakutendea wema na wewe nilipe wema'. Hii ni kwa sababu, anayetenda wema anatarajia kulipwa wema. Kwa kawaida wanaotendeana wema ni watu wanye mapenzi baina yao. Kwani mapenzi ni huruma ya kutendeana wema maishani.

Ama, leksimu ndege, kwenye msemo (2), asili yake ni kiumbe mwenye miguu miwili, manyoya na mbawa. Kiumbe huyu hazai bali hutaga mayai na kuangua vifaranga. Wapo ndege wenye uwezo wa kupaa angani na wapo waso na uwezo huo. Wapo wenye rangi na sura za kuvutia kama kasuku, manga, na wapo wenye rangi na sura za kuogofya kama bundi. Aghalabu, binadamu huweka tunduni ndege wenyekuvutia kwa ajili ya kujifurahisha nafsi zao. Hivyo, fasili ya msemo huu, ni kwamba mfugaji anamwambia ndege anayemfuga kwenye tundu, aendelee kukaa humo humo (tunduni), kwani akiondoka, atamsikitisha.

Maana ya matini yaweza kupatikana kwa kutumia msimbo wa ishara. Msimbo huu unaeleza kwamba maana ya ishara inatoka kwenye jamii na si kwenye mitambo ya kufyatulia maana. Hivyo, kwa mujibu wa jamii ya Wazanzibari ndege ni ishara inayorejelea mke. Mke amefananishwa na ndege anayefugwa kwa sababu ya uzuri wake. Vile vile, mke ni pumbazo na burudisho la mumewe. Kwa upande mwingine, tunduni, kwa maana ya matini ni nyumbani au ndoani. Hivyo, maana matini ya msemo huu wa kwenye kawa ni tulia katika ndoa, nisipatwe na huzuni.

Nao moyo katika msemo (3) nina moyo sina jiwe mengine nimpunguziwe unaweza kuchambuliwa kwa maana ya asili kama kiungo miongoni mwa viuongo vya mwilini. Kiungo hicho kina kazi ya kusafisha na kusafirisha damu sehemu nyingine mwilini. Moyo ndio mtambo unaoendesha uhai wa viumbe hai. Moyo ukizimika na uhai umezimika. Aidha moyo ni rahisi kuathiriwa na hisia nzuri au mbaya. Moyo hupata maradhi/mshtuko unaoweza kusababisha kifo unapopata habari mbaya au za kuhuzunisha. Ama, jiwe ni kitu kigumu, kisicho hai na hivyo hakina hisia yoyote; likiudhiwa, likisimangwa, lipigwa litabaki kuwa vilevile lilivyo. Halishtuki wala haliwezi kupoteza uhai (tangu hapo halina uhai)

Kutokana na maana hiyo ya asili tunaweza kupata maana matini ya nina moyo sina jiwe ni ninaumia kwa mnayonifanyia, na maana matini ya upande wa pili mengine nipunguziwe ni nioneeni huruma. Msemo huu hutumika zaidi pale mtu anapoona watu wanamsakama kwa maneno, wanamsingizia uovu, akifanya jema haonekani. Hivyo, hutumia msemo huu kupaza sauti yake kuumbea moyo wake upatiwe afueni.

Ishara nyingine ni paka katika msemo wa (4). Neno paka laweza likawa na maana nyingi lakini katika msemo huu paka aliyekusudiwa ni paka mnyama. Paka ni mnyama afugwae na mwanadamu. Ni mnyama mpole na husaidia kukamata panya na hivyo kuondoa viumbe viharibifu katika nyumba. Hata hivyo, paka hawezi kumsaidia 
mchungaji wake anapoumwa, hawezi kumsaidia kazi za ndani, hawezi kuzungumza nae maneno ya kumfariji anapokuwa na huzuni wala hawezi kushauriana nae lolote; kwani paka hasemi. Ama, majirani ni watu wa karibu katika makaazi. Ndiwo watu wa kwanza kuweza kujua shida za nyumba ya pili na kutoa msaada.

Katika msemo huu ni bora kufasili maana matini moja kwa moja kwani haiyumkiniki mtu kuona bora kuishi na mnyama kuliko na binadamu mwenziwe. Lazima kuna jambo. Makala imegundua kwamba jirani akiamuwa kuwa mbaya anaweza kuwa mbaya kwelikweli kuliko paka mwizi wa vitoweo. Jirani anaweza akatumia ukaribu wake, kuaminiwa kwake na kutegemewa anakotegemewa kufanya atakalo kwa jirani yake. Mpaka mtu anapotahamaki kishafanyiwa ufisadi wa maana shindwe hata kujipapatua. Ndio maana kuna usemi mwingine usemao kikulacho ki nguoni mwako. Hivyo, maana matini ya msemo huu ni kuwa si kila anayetegemewa huwa ni mwema.

Msimbo wa Kihemenetiki

Misemo iliyokuwa na matendo ya binadamu yaliyotendwa kiishara ni hii ifuatayo:

1. Funika vifunikie mlaji afurahike

2. Ni zako zabibu kula taratibu

3. Kitunze usikitupe kitaokotwa ujute

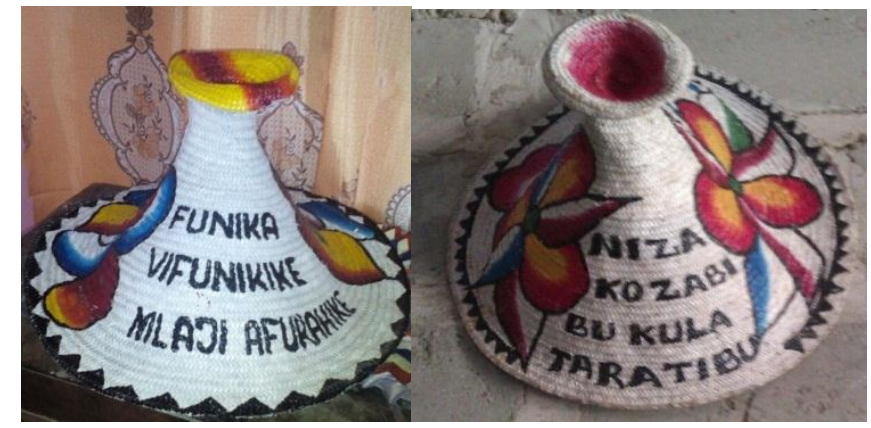

\section{Chanzo: Mtafiti (2016)}

Msemo (5), funika vifunikike mlaji afurahike una matedno yanayoashiria kazi zote tatu za kawa. Kazi hizo ni kufunika, kufanya mawasiliano na kupamba nyumba. Hivyo maana yake ilichambuliwa kwa kutumia msimbo wa kihemeniki unaoshughulikia fasili za ishara za kimatendo yanayotendwa na binadamu. Kwanza kabisa funika katika maana ya asili, ni kukisitiri kitu kisionekane, kihifadhike, kitunzike. Ama neno vifunikike lina maana huko kufunika kufanyike kwa umakini ili tendo la kuhifadhi likamilike kama lilivyokusudiwa. Ufunikaji ukiwa umekidhi sifa za kufunika basi mlaji hufurahika. Hapa neno mlaji linatuashiria kwamba kinachotakiwa kufunikwa si gari wala vyombo wala chochote kingine bali ni chakula. Na hii; kufunika chakula ni kazi ya kawa. Hivyo, maana ya asili ya semi hii ni mlaji hufurahika anapokuwa na uhakika kuwa chakula alacho ni nadhifu; safi na salama.

Kama ilivyo kanuni ya mawasiliano, maana haipatikani tu, kwa kufasili maneno bali pia kuchunguza matukio yaliyosababisha tamko fulani kutumika. Nani atumiaye tamko hili na wapi linapotumika. Hivyo, kitendo Funika ni ishara yenye maana tulia, jisitiri, jihifadhi. Naye mlaji katika maana batini ni mke/mume. Kwa mkabala huo, maana batini ya msemo nzima ni tulia, ukweli wa kutulia katika ndoa ili mume/mke/ awe na amani.

Katika msemo wa (6) kabla ya kuelezea maana ya kihemenetiki ya kitendo kula taratibu ni bora kwanza tuchambue leksimu zabibu. Hii itatusaidia kujua ni kwanini linatakiwa kuliwa taratibu. Kwa asili Zabibu ni tunda, kama lilivyoelezewa hapo juu kwenye msemo usemao kula zabibu ulete majibu. Maana hiyo hiyo ya asili ya zabibu ndiyo inayotumika katika msemo ni zako zabibu kula taratibu. Kwa maana matini ya zabibu katika msemo ni zako zabibu kula taratibu ni mapenzi au mke. Zabibu zimefananishwa na mapenzi/mke kwa sababu tunda hilo lina haiba ya kuvutia na hutuliza macho na nafsi ya mtazamaji. Vile vile, ni laini, rahisi kuvurugika lisipobebwa kwa makini. Vivyo hivyo, mapenzi/mke ni rahisi kuvunjika yasipoenziwa. Hivyo, mapenzi/mke ni zabibu na si fenesi. Hali hii ya mke kufananishwa na tunda pia imeleezwa na Mlingi (2013:40 -41) alipokuwa akifafanua matumizi ya taswira katika msemo wa kwenye kawa unaosema kula pole pole nanasi hili ni lako.

Kwa hiyo, kitendo Kula taratibu, kwa maana ya asili ni kutia chakula mdomoni, kukitafuna na kumeza bila kufundia. Na ilivyo kikawaida zabibu zinatarajiwa kuliwa; zitiwe mdomoni, zitafunwe na kisha zimezwe. Hata 
hivyo, msimbo wa Kihemenitiki wa NS unaeleza kwamba kuna matendo ya binadamu ambayo hutendwa kwa kuashiria jambo fulani (Barthes 1986, Wanjiku 2006, Taib 2014). Katika msemo huu kula taratibu ina maana kuenzi au kuacha papatiko la nafsi. Msemo huu unafanana na ule wa kidigo isemayo Avi umacheyo kufundira n'kwani (upo peke yako kufundia ni kwanini)

Hivyo, maana ya asili ya msemo ni zako zabibu kula taratibu ni usifanye papara zabibu (tunda) ni mali/milki yako. Hivyo, kula polepole (hakuna atakayekunyang'anya). Lakini maana batini ya msemo huu ni mke/mume ni wako, na mtu kitu chake anatakiwa akitunze. Hivyo, kula taratibu ni kumuenzi mke/mume. Maana ya pili iliyopatikana katika msemo ni zako zabibu kula taratibu ni upendo (wangu) ni mali/milki yako usiwe na wasiwasi kwamba atakuja mtu mwingine akunyang'anye. Msemo mwingine wenye maana sawa na huu ni ule unaosema ni lako zaituni wacha tafrani.

Hali hii ya kumchukulia mwanamke kuwa ni tunda imeelezwa na baadhi ya watafiti kuwa ni udhalilishaji wa mwanamke (Mlingi 2013, Mkonwa 2014). Matokeo hayo yametokana na nadhari walizotumia. Mlingi alitumia nadharia ya Mwitiko wa Msomaji na Mkonwa alitumia nadharia ya Unisai, Hivyo, nadharia aliyotumia Mlingi ilimpa uhuru msomji kufasili kauli vile anavyoona yeye. Nadharia ya Unisai aliyotumia Mkonwa inapinga mfumo dume hivyo mwanamke hachukuliwa kama kitu laini na anayehitaji kutunzwa ni kumdharau na kumdhalilisha.

Ama, kitendo Usikitupe, katika msemo wa (7) kwa maana ya asili ni usiweke kitu mahala pasipofaa, hasa kwa kukirembea/kukirusha mfano jaani au kwenye pipa la taka. Neno usikitupe ni kitenzi kilichotumika kiishara. Ishara hiyo, ina maana ya usikifuje, badala yake kienzi/kihifadhi. Kitaokotwa, katika maana ya asili ni kitachukuliwa kutoka hapo kilipotupwa. Maana ya kimatini ya neno kitaokotwa ni wengine watakichukua kwa kujua kwamba mwenyewe hakitaki. Hivyo, maana kamili ya msemo kienzi usikitupe kitaokotwa ujute ni kienzi usikidharau, la sivyo, wengine watakichukua ubaki na majuto. Kwa mantiki hiyo, kutupa ni kutokukishughulikia kitu vile inavyotakikanwa. Mathalan, mtu anaweza akasema fulani amewatupa wazee wake au ameitupa aila yake, akiwa na maana hawaangalii kwa hali wala kwa mali.

\section{Msimbo wa Matukio}

Kuna baadhi ya misemo ya kwenye makawa yenye matukio yanayosababishana. Misemo hiyo ilichambualiwa kwa kutumia msimbo wa matukio. Misemo hiyo ni:

1. Niweke nikae niwe dawa nikufae

2. Ikiwa ndio tabia siwezi kuvumilia

3. Tulia tuishi wazuri haweshi

Niweke nikae katika maana yake ya asili ni nitie mahala niwe katika hali ya kitako. Niwe dawa nikufae maana yake ya asili yaweza kupatikana vizuri zaidi kwa kuchambua neno moja baada ya moja. Dawa, ni kitu kinachotumika kutibu maradhi. Dawa yaweza kuwa ya kupaka, kuoga, kujifukiza, kunywa au kumeza. Mbali na hayo, dawa yaweza kuwa tamu, chungu au isiwe na ladha kama maji. Dawa, pia yawezakuwa na harufu. Na harufu hiyo yawezakuwa nzuri au mbaya. Vyovyote iwavyo, dawa kazi yake ni kutibu. Nikufae maana yake nikutumikie, unitumie. Hivyo, maana ya asili ya msemo huu ni nitie mahala nipate kukaa (kitako) ili nikutibu.

Kwa mujibu wa msimbo wa matukio (Barthes 1987) unaoeleza kwamba matukio hufuatana na kusababishana katika kujenga maana ya ziada. Niweke nikae inaweza kufasili kwamba awali, ili mtu akae, ni lazima aridhike. Na ili aridhike ni lazima afanyiwe mambo yenye kuridhisha. Hivyo, niweke nikae, ina maana nifanyie mambo ya kuniridhisha, ili nitulie. Ama niwe dawa nikufae, inaendeleza maana iliyopatikana katika sehemu ya mwanzo ya msemo huu. Yaani mtu akifanyiwa wema atatulia/hataondoka/hatamuwacha mwenza, kisha atakuwa mwenye manufaa. Niwe dawa nikufae, na wala si, niwe sumu nikudhuru. Hivyo, msemo hiyo ina maana nifanyie wema ili niwe na manufaa kwako.Maelezo kama haya yalitolewa na Salim (2015:60) alipokuwa akieleza dhima za msemo unaosema ukitaka nitulie maudhi usinifanyie.

Msemo (8) hapo juu unaanza na sharti, kwa kutumia neno ikiwa. Sharti hili linatoa ishara kwamba kuna jambo fulani lazima lifanyike kwanza, ndipo jambo/mambo mengine yafuate. Kwa mantiki hiyo maana ya msemo huu yaweza kupatikana vizuri kwa kutumia msimbo huu wa matukio unaosisitiza mfuatano wa matukio na jinsi yanavyosababishana (Barthes 1986). Sharti linalotolewa katika kauli hii linahusu tabia. Mwanzo wa kauli ikiwa ndio tabia haudhihirishi waziwazi tabia hiyo ni ipi. Lakini, mwisho wa kauli unasema siwezi kuvumilia unatoa ishara 
kushindwa kuvumili kunasababishwa na tabia kuwa mbaya. Hivyo, mfuatano wa matukio katika msemo huu unabeba maana kwamba tabia mbaya haivumiliki.

Waswahili husema mazoea hujenga tabia. Mtu akijipamba kwa tabia nzuri hupendwa na watu na akijisiriba kwa tabia mbaya huchukiwa na watu. Katika msemo huu Ikiwa ndio tabia siwezi vumilia kinachodhihirika ni mazoea mabaya. Na mazoea mabaya hujenga tabia mbaya. Tabia mbaya huleta maudhi. Kutokana na ufasili huo yumkini maana ya msemo wa kwenye kawa ikiwa ndio tabia siwezi kuvumilia ni, ukiendelea na tabia yako (mbaya) sitostahamili. Kuchoka kuvumilia kunaweza kushadidiwa na uchambuzi wa Mkonwa (2014:65) aliyechambua msemo ikiwa ndio tabia siwezi kuvumilia kwa kusema uvumilivu una kikomo ... Vile vile, kuna msemo mwingine unaosema nastahamili dhiki kuliko maudhi.

\section{Msimbo wa Kirejelezi ifuatayo: \\ 1. Harusi ni jambo la kheri \\ 2. Vyako n'vyako vyako na wenzio \\ 3. Karibuni wageni \\ 4. Pweza kamwambie ngisi yasokuhusu usiyadadisi}

Maana za misemo ya kwenye makawa zilizopatikana kwa kurejelea tajriba na utamaduni wa Wazanzibari ni hii

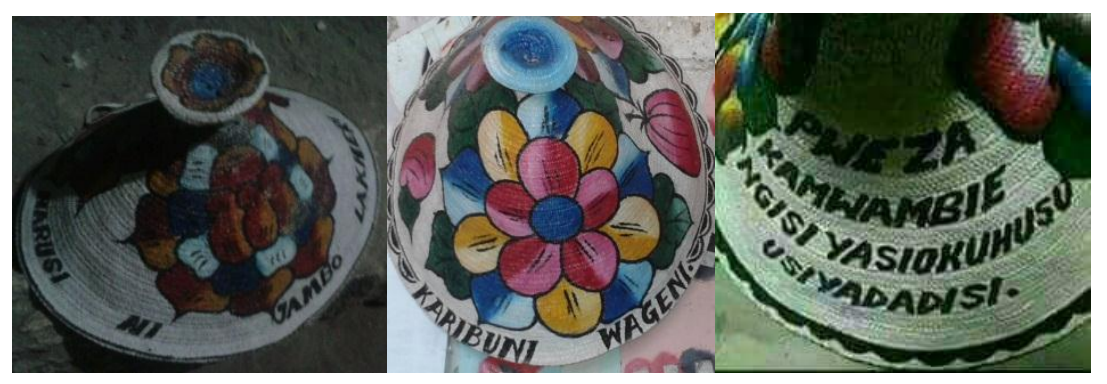

\section{Chanzo: mtafiti (2016)}

Maana ya asili iliyopatikana kutika msemo harusi ni jambo la kheri ni sherehe za mafungamano ya kisheria kati ya mwanamume na mwanamke katika ndoa kuwa ni jambo zuri. Harusi ni sherehe baada ya kufunga ndoa kwa wawili (mwanamume na mwanamke), wanaopendana. Kupendana ni jambo zuri. kwa sababu ni kitendo kinachotarajiwa kulete hali ya kuleana kwa huruma na kuliwazana ili wapendanao wapate utulivu wa nafsi zao. Hivyo, neno kheri limetumika kiishara zaidi na linalipatia msemo huu maana nzito zaidi.

Kheri hiyo inatokana na ukweli kwamba ndoa ina manufaa kwa jamii nzima. Miongoni mwa manufaa hayo ni kuunga udugu, kuongeza ukoo kwa njia halali, wajane kupata stara, watoto viate au yatima kupata tena baba ama mama wa kulelewa naye katika familia. Hivyo, mbali na maharusi wenyewe kupata utulivu wa nafsi, jamii yote kwa jumla inanufaika.

Kwa kutumia msimo wa urejelezi wa nadharia ya Semeotiki (Barthes 1963, Taib 2014) mtafiti alibaini kwamba tukio la harusi ni jambo linalorejela furaha na fakhari kwa wahusika. Umuhimu huo unaona na falsafa ya maisha ya ndoa ya jamii ya Wazanzibari. Jamii ya Wazanzibari inaitukuza harusi kwa kuwa ni sherehe ya maana. Harusi inawatakasa wazazi na mtoto pia. Mtoto akioa/olewa, anaepukana na vishawishi ambavyo vingeweza kumsababishia apate watoto nje ya ndoa. Katika jamii hiyo, watoto wa nje ya ndoa, ingawa wapo, lakini si jambo linalopendwa (Yahya-Othman 1997). Hivyo, watu hufurahi kukaa vikao kuzungumzia mipango ya ndoa kuliko kuzungumzia uja uzito wa nje ya ndoa.

Maana matini ya karibuni wageni (13) ni hapa ni kwenu. Mgeni ni mtu asiyezoea jambo au mahali fulani. Mtu anaweza akaitwa mgeni wa kutunga mashairi (mwanagenzi) au mgeni wa mji. Lakini "mgeni wa heshima" si kwamba hajui kuheshimu au kuheshimiwa la hasha. Mgeni wa heshima ni mgeni anayeheshimiwa pengine kuliko wote walioalikwa. Mkorogano huu wa kimaana huwafanya viongozi wengine wakatae kuitwa mgeni wa heshima. Mathalan, aliyekuwa Mkuu wa Mkoa wa Magharibi Maalim Abdalla Mwinyi.. Aidha, mtu na mtu pia waweza wakawa na ugeni baina yao. Ndipo mtu husema sura yake ni ngeni machoni mwangu. Hivyo, mtu wa namna hiyo anatolewa wasiwasi wa kujisikia hayupo kwao kwa semi karibuni wageni. 
Vyako n’vyako vyako na wenzio (12) ni msemo ulioandikwa kwa Kiswahili cha Kitumbatu. Kwa Kiswahili cha shuleni lilisomeka kama vyako ni vyako vyako na wenzako. Maana ya msemo huu, ni vitu vyako, ni vyako, lakini hivyo vyako na wenzako pia vinawahusu. Maana ya matini ya, vyako n’vyako vyako na wenzio, ni usiwe mbinafsi.

Maana hiyo imerejelea muktadha wa maisha ya jamii ya Watumbatu. Msimbo wa Kirejelezi wa Barthes (1964, 1977, 1981, 1984, 1986) uanoelekeza kurejelea utamaduni na tajriba ya maisha ya jamii inayohusika na kazi inayohakikiwa. Makala hii ilitumia msimbo huo na kurejelea maisha ya Watumbatu ambapo ilibainika kwamba Watumbatu wana tabia ya kuishi nje ya kisiwa chao kwa kipindi kirefu cha mwaka. Hata hivyo, wazee huhakikisha vijana hawapotelei huko wanakokwenda kutafuta maisha. Hivyo, wanatumia fasihi simulizi kama msemo huu kuwafanya wakumbuke kwao. Na inapotokea kijana wa Kitumbatu kuishi nje ya kisiwa chake, basi ni katika maeneo ya karibu ya kisiwa hicho, mfano Mkokotoni, Mkwajuni, Fukuchani, Kidoti, Nungwi, Kinyasini, Bumbwini, Chaani na popote Utumbatuni.

Maana asili ya msemo (13) Pweza kamwambie ngisi yasiokuhusu usiyadadisi ni samaki aina ya pweza anatumwa akambwambie samaki aina ya ngisi asifuatilie maisha ya watu. Ama, maana matini ya semi hii ni kamwambie jirani yake asifuatilie mambo yasiyomuhusu. Pweza na ngisi ni viumbe vya majini, hususan baharini, ni majirani. Salamu akipewa pweza, ngisi zitamfika. Vile vile, salamu akipewa ngisi, pweza zitamfika. Aidha, salamu hizi kapewa pweza ampelekee ngisi na si ngisi ampelekee pweza. Mbali na kutaka kuleta usanaa wa urari wa vina pia tabia za wawili hao ndizo zilizosababisha mmoja wao kuwa mpeleka salamu na mwingine mpokeaji. Kitabia, pweza hachezi mbali na makaazi yake (mwambani). Ngisi huranda sana baharini tena kwenye kina kirefu chenye maji safi zaidi ya pweza. Kutulia kwa pweza kuna maana yeye si mbea (anaejihusisha na ya wengine) ilhali kuranda kwa ngisi kuna maana ana fursa nzuri ya kupekuwa maisha ya wengine. Hivyo, moja kwa moja anachukuliwa kuwa ni mbea. Hapa, msimbo wa urejelezi (Barthes 1987) unatuelekeza kwamba, urejelezi huu wa tajriba ya maisha ya pweza na ngisi unasaidia kupata maana ya semi pweza kamwambie ngisi yasokuhusu usiyadadisi

\section{Msimbo wa Ki -ishara}

Msimbo huu ulibeba misemo ya kwenye makawa iliyokuwa na ishara zilizotoa muelekeo wa kutokea kwa jambo Fulani.

1. Yarabi tupe salama ya duniani na kesho kiama

2. Mola ibariki ndoa wasipate doa

3. Nisipokuona machoni sina furaha moyoni

4. Innallah maaswabiriin
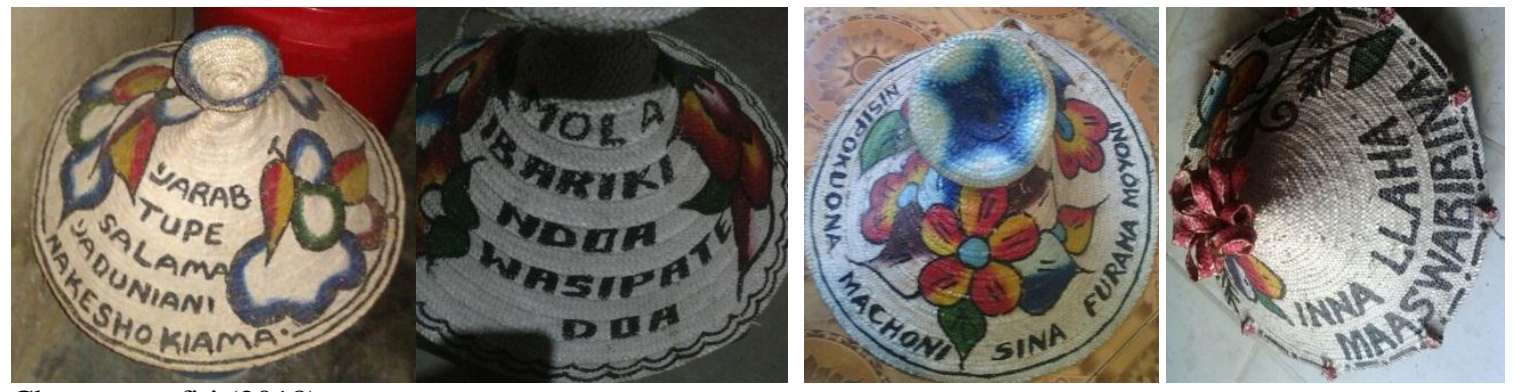

Chanzo:mtafiti (2018)

Katika maana asili (15), Yaa Rabb tupe salama ya duniani maana yake ni Mola Mlezi tupe amani ya nafsi kutoka kwako katika sayari hii ya dunia. Ama na kesho kiama maana yake ni siku ya kesho inaitwa kiama (kama ambavyo inaweza kuwa kesho Jumatatu). Kwa upande wa maana matini, Ya Rabi tupe salama ya duniani maana yake ni Mmiliki wa salama tuepushe na misukosuko na balaa katika maisha ya kila siku. Ama, na Kesho kiama inatoa muelekeo kwamba kuna siku viumbe watasimimishwa kwa ajili ya kuhukumiwa. Neno kiama lina asili ya neno la Kiarabu 'kiam' lenye maana ya kisimamo Siku hiyo, waja wote watasimama mbele ya Mwenyezi Mungu kwa ajili ya kuhukumiwa kutokana na matendo waliyotenda humu duaniani.

Mola ibariki ndoa wasipate doa ni Mlezi wa Viumbe ipe baraka ndoa ili wanandoa wasipate uchafu. Hii ni maana asili. Doa ni alama inayoingia mahala isipotakiwa kuingia na ikawa tabu kuiondoa. Doa laweza kuwa la rangi, maembe, madafu, damu. Maana matini ya Mola ibariki ndoa ni Mlezi wa Viumbe ifanye ndoa iwe yenye manufaa. 
Wasipate doa, kipande hiki kinawakusudia wanandoa, kwamba wasipatwe na kasoro, au madhara, au matatizo katika ndoa yao.

Ingawa dua inaombewa ndoa lakini walengwa wa dua hiyo ni wana ndoa. Ndio maana msemo unamalizikia na wasipate doa, ikionesha kuwarejelea wana ndoa, na wala haimalizikii na isipate doa kwa kuirejelea ndoa. Hii ina maana kwamba ndoa ikipata doa watakaoathirika ni wana ndoa. Kwa hiyo, ndoa ikiwa na baraka inatoa ishara kwamba wanandoa watakuwa na amani, mafanikio, furaha, utulivu na kila linalotarajiwa kuletwa na baraka.

Nisipokuona machoni sina furaha moyoni kwa maana matini ni kwamba macho yasipoona yakipendacho, moyo hukosa utulivu. Macho huona. Hakumalizikii kuona tu bali kilichoonekanwa hupelekwa moyoni. Moyo hujenga hisia kutokana na kile kilichoonekanwa na macho. Hivyo, mtu akiona jambo zuri, moyo hufurahi. Kinyume chake, macho yakiona jambo baya, moyo huchukia au huhuzunika. Kwa maana hiyo hisia zijengwazo moyoni hutegemea kile kilichoonwa na macho; moyo pekee haujitoshelezi katika kujenga hisia. Hivyo, msemo huu una maana, apendaye huhuzunika asipomuona yule ampendaye. Hutamani amuone machoni kila wakati na asipo muona hukosa furaha moyoni. Kwa kufanya hivyo penzi litakuwa imara zaidi. Salim (2015:45) anaeleza kwamba si vizuri mtu kuwa mbali na mpenzi wake labda kuwe na dharura ya kufanya hivyo. Mtafiti alieleza hayo alipokuwa akichambua msemo wa mkoba unaosema nisipokuona dakika roho hunihangaika

Innallah maaswaabiriin tarajuma ya msemo huu kwa lugha ya Kiswahili, ni hakika ya Allah, Yu pamoja na wenye kusubiri. Ama, maana yake ya asili ni kwamba Allah hukaa na wenye kusubiri. Mwenyezi Mungu hukaa na wenye kusubiri maana yake ni kwamba Mwenyezi Mungu Amewapenda na Ameridhika nao. Kwa maana hiyo kusubiri ni jambo linaloashiria kutokea kwa upendo na Mwenyezi Mungu. Hivyo maana matini ya msemo huu ni Allah Anawapenda watu wenye subira.

Kusubiri ni suala linaloendana na imani kwa Mungu kama alivyoeleza Mlingi (ameshatajwa) alipokuwa akichamba msemo kila mwenye kusubiri yupo na Mungu. Msemo huo kwa mujibu wa mtafiti huyo unabeba dhamira ya imani kwa Mungu. Dhamira hiyo ilipatikana kwa kutumia nadharia ya mwitiko wa msomaji.

\section{Hitimisho}

Makala yamebaini kuwa kawa si kifaa cha kufunikia chakula tu, bali pia ni ishara ya stara, umaridadi na njia ya mawasiliano. Aidha, misemo inayoandikwa kwenye makawa inabeba maana zinazobainisha busara, hekima na maadili ya Wazanzibari. Isitoshe, ipo misemo iliyotumia ishara ambazo zinabeba maana asili na maana matini. Vile vile, ipo misemo ya kwenye makawa ambayo haikutumia ishara. Misemo ambayo haina ishara ina maana asili tu. Hata hivyo, maana hasa ya misemo hiyo ni ile iliyofasiliwa kutoka katika maana matini. Hii ni kwa sababu misemo hiyo hutumia ishara ambazo zinafahamika vizuri zaidi kwa kurejelea matukio katika maisha ya watumiaji wenyewe. Hivyo, kifaa hiki kinafunika utamaduni wa Wazanzibari ili kinahifadhi.

\section{Marejeleo:-}

1. Al-Farsy, A.S.(1984). Quran Takatifu. Nairobi: The Islamic Foundation.

2. Ali, A.Y. (1973). The Glorious Holy Kur'an, Translation and Commentary. Tripoli: Islamic Call Society.

3. Amour, S (1994) "Hotuba ya Rais wa Zanzibar katika Ufunguzi wa Mkutanao wa kwanza wa Kimataifa juu ya Historia na Utamaduni wa Zanzibar. " katika Historia Fupi ya Zanzibar, Idara ya Nyaraka, Makumbusho na Mambo ya Kale, (Wh) Khamis na Omar. Uk xi-xv

4. Anderson, L., \& Trudgill, P. (1990). Bad Language. Oxford: Basil Blackwell Ltd.

5. Aoron, D. (1995) Hands full of Meaning. Clarendon: Oxford Arabiyyah. Fraser, B. (1996). "The Domain of Pragmatics". Katika Language and Communication.

6. Ashipu na Amende (2013). "Proverbs as Circumstantial Speech Acts", katika Research on Humanities and Social Sciences. Vol 3, No. 7 www.jiste.org

7. Beck, R. M (2001) “Ambiguous Signs: The Role of the Kanga Names as a Medium of Communication” katika AAP 68(2001) Swahili Forum VIII. (uk 157-169)

8. Barthes, R. (1977) "Image, Music and Text." Richard Howard (tran), katika David H. Richter.

9. The Critical Tradition: Classic Texts and Contemporary Trends. New York: Bedford Books.

10. (1981) "Theory of the Text." Ian MacLeod (tran), katika R. Young, Untying the Text. London: Routledge.

11. Finnegan, R. (1970) Oral Literature in Africa. Oxford University Pess. Nairobi.

12. Innis, R.E (1985) Semiotic. London: Indiana Universit Press. 
13. Kipacha, A. (2014) "Semi za Vyombo vya Usafiri: Divai Mpya?" katika www.sciencedirect.com Social and Behavioral Sciences 136 (2014) pp 318-321

14. Kimani, H. W., (2006) Uwiano wa Picha na Matini katika Mabango ya Matangazo ya UKIMWI: Mtazamo wa Kisemiotiki. Tasnifu ya Shahada ya Uzamili, Kiswahili, Chuo Kikuu cha Kenyatta.

15. Mauya, B. (2006). Semi: Maana na Matumizi. Dar es Salaam: Taasisi ya Uchunguzi wa Kiswahili, Chuo Kikuu.

16. Mlingi, S.J. (2013), Dhima ya Semi za Kawa katika Jamii ya Watu wa Pwani, Tasinifu ya M.A Kiswahili, Chuo Kikuu Huria Cha Tanzania.

17. Mkonwa, A. (2014), "Usawiri wa Mwanamke katika Misemo iliyoandikwa kwenye Vipepeo -Tanga," Tasnifu ya Uzamili, Chuo Kikuu cha Dar-es-salaam.

18. Mwamzandi, I. Y., (2013) “Metafizikia ya Kuwapo katika Methali za Waswahili: Uhakiki wa Kiudenguzi” katika Kioo cha Lugha, Juz. 11 (Wh. Omar Sh. Na Peterson Rh.) uk 1- 13

19. Ngozi, I. S. (2001) An Approach to Literature and Literary Criticism Based on Islami Principles and Aesthetics.

20. Tasnifu ya $\mathrm{PhD}$ (Literature) iliyowasilishwa katika Islamic University In Uganda (Haijachapishwa)

21. Salim, A. (2015), "Kuchunguza Dhima za Semi zilizoandikwa kwenye Mikoba Kisiwani Pemba," Tasinifu ya Uzamili, Chuo Kikuu Huria cha Tanzania,

22. Sengo, T.S.Y. (1987), Fasihi Simulizi, Sekondari na Vyuo: Dar es Salaam: Nyanza Publications Agency.

23. (2008) Tafakuri ya Utu na Uhai, Huda Publishers: Dar-es-salaam

24. (2009), Sengo na Fasihi za Kinchi. Dar es Salaam: AKADEMIYA.

25. Syzdykov, K., (2018 May,15) Contrastive Studies on Proverbs www.sciencedirect.com Taib, A.H (2008).

26. "Mkabala wa Kiislamu katika Uchanganuzi na Uhakiki wa Fasihi ya Kiswahili", katika Ogechi N.O na wenzake (wah) Nadharia katika Taaluma ya Kiswahili na Lugha za Kiafrika. Eldoret Kenya: Moi University Press.

27. (2014) Taashira katika Nyimbo za Harusi za Wazanzibari. Tasnifu ya Uzamivu.

28. Chuo Kikuu cha Egerton, Nakuru, Kenya.

29. TUKI, (2004). Kamusi ya Kiswahili Sanifu; Toleo la Pili. Dar es Salaam: Taasisi ya Uchunguzi wa Kiswahili. 